\title{
Coverage-Aware Protocols in Wireless Sensor Networks: A Review
}

\author{
Jahangir Khan ${ }^{1}$, Khalid Mahmood ${ }^{2}$, Ansar Munir Shah ${ }^{3}$, Babar Nawaz ${ }^{4}$, Mahmood ul Hassan ${ }^{5}$ \\ Department of Computer Science, \\ IIC University of Technology, \\ Phnom Penh, Cambodia
}

\begin{abstract}
Coverage plays a vital role in wireless sensor networks (WSNs), since it is used as one of the important measure to achieve the performance of the sensor network. The sensor nodes in WSN have limited power and energy resources. So, energy efficiency is an essential factor that should be considered along with coverage while designing the coverage protocols. During the past few years, many efforts have been made by the researchers on designing different coverage-aware protocols. Different coverage-aware protocols may impose different ways to solve the coverage and efficient utilization of energy among sensor nodes. In this paper, we present a review on coverage-aware protocols by highlighting their functionalities.
\end{abstract}

Keywords-Wireless sensor network; coverage area; energy efficiency; coverage protocols

\section{INTRODUCTION}

In recent years, wireless sensor networks (WSNs) have been implemented in many application areas like wild fire detection, habitat monitoring, battlefield, industrial, agricultural and medical etc. [1]. In WSN, large numbers of sensors are deployed in the field of interest. The deployed sensors are low cost, small in size, and have limited power and communication capabilities. Moreover, sensor nodes communicate with each other and can cover the anticipated field of interest. In WSN, sensor nodes have the capability to sense the activities in an area of interest, process them, and can forward them to the base station [2]. The most essential factor to install an efficient sensor network is to search the best node installment schemes and efficient topology approaches [3] [4]. To manage the connectivity coverage is very essential for a sensor network.

Mostly sensors are supplied with limited energy resources. In particular applications, nodes are deployed in rigid surrounding, from where it is tough to recharge or change the batteries. Without any predefined planning, nodes are randomly placed in a desired region. Once nodes are deployed, they can automatically configure the network. In sensor network applications, numerous sensors are placed in the territory. Hence, there might be a chance that one or more sensor nodes are tracking a covering region. So, the data sensed by more than one sensor nodes have a small amount of redundancy. The optimal approach is to deploy lowest number of sensors in an area to get full coverage.

The life cycle of a sensor network can be determined by the time period in which a network is capable to achieve the sensing and communication task with the base station (BS) [5].
At the same time, a few sensor nodes may fail due to the hardware breakdown or power deficiency. Meanwhile there might be a chance of deploying more nodes to sustain the suitable coverage degree. In addition, when there is no more coverage possible and sensing voids appear then the coverageaware protocols need to manage the sensing voids. The coverage redundancy appears in WSN, if more than one sensor nodes are sensing the same region which would result in more consumption of energy. Due to the limited energy resources in WSN, it is essential to detect the active redundant nodes and put them in OFF state unless they are needed to be in ON state. Due to the small power battery, we must use the sensor nodes in an efficient way so as to maximize the network life cycle [6].

The biggest key issue raised recently is how to deal with the coverage. Based on the object covering, the coverage issue can be categorized into path, region and target coverage. Each point in the region must be tracked by minimum number of nodes. To achieve the best coverage, the nodes should be placed with particular amount in order to cover the complete region. Therefore, coverage-aware protocols are needed to be designed in such a way that they can achieve full coverage with efficient utilization of power and communication resources. This paper provides a comprehensive review on coverageaware protocols.

Rest of the paper is organized as follows. Section 2 highlights the coverage and connectivity problems based on the energy consumption and deployment strategies. Section 3 briefly provides an overview on coverage-aware protocols based on comparison study. Finally the section 4 concludes the review article.

\section{LITERATURE REVIEW}

Wireless sensor network is configured in an area of interest to provide the best coverage along with efficient utilization of energy. The proposed schemes for coverage in a wireless sensor network must consider several factors. The capacity of sensors should be reviewed. Mostly researchers emphasize on only one deployment model. Every sensor has a specific covering capability to cover an object in the deployed field. A node can cover a small range of field. Hence, in a WSN various sensing models can be composed according to the needs of the environment.

Some sensor nodes have uniform communication ranges; many sensors' radio transceiver has the capability of altering 
communication power continuously to acquire various transmission ranges. After the deployment of sensor nodes either distributed or centralized algorithm is run to find the sufficient coverage in the field. The coverage algorithm executes in a center node. Data from entire sensor nodes requires to be forwarded to central node. In distributed approach, the coverage algorithm is run in WSN which depends upon data from various nodes.

Mostly a huge number of sensors are placed in a territory to be supervised. In WSN, numbers of sensors are generally more than mobile-adhoc network [7]. In a WSN, placement of nodes can generally be classified as a dense deployment or a sparse deployment. In dense deployment, large numbers of sensor nodes are scattered in the field of interest. On the other hand in sparse deployment, few nodes are scattered in the field of interest. The deployment of sensor nodes in a WSN can be deterministic or random as shown in Fig. 1 and Fig. 2. In random approach it is compulsory to place extra nodes to get the full coverage.

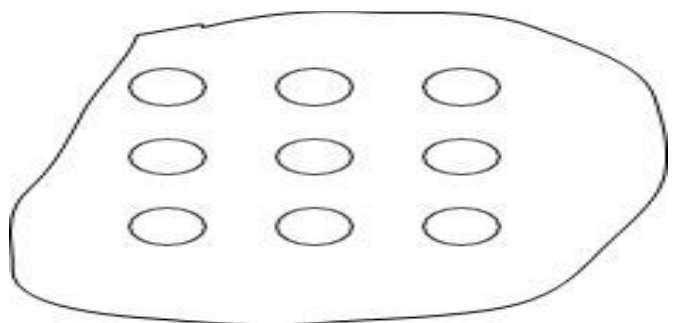

Fig. 1. Deterministic Deployment of Nodes.

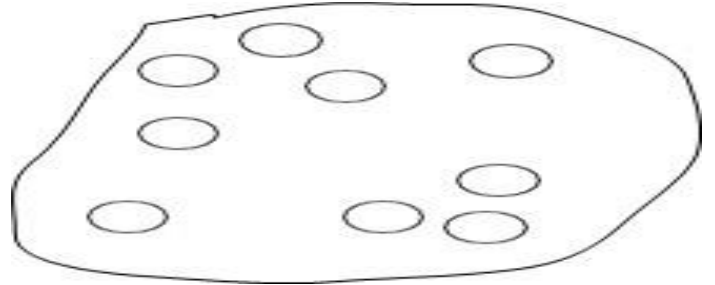

Fig. 2. Random Deployment of Nodes.

The following section provides a brief review on coverageaware protocols for WSN.

\section{A. Low-energy Adaptive Clustering Hierarchy (LEACH)}

The LEACH protocol is a cluster-based scheme for small sensor networks which integrates the concepts of media access and energy- efficient cluster based routing that is combined with application specific data collection in order to get the application perceived quality, latency and good performance based on life cycle of the network [8]. The objective of LEACH is to switch off the nodes that are non-head nodes as much as possible. The function is distributed into periods and each period contains a steady phase and set-up phase. At the same time, set-up phase selects the cluster heads and every node gets a cluster by selecting the cluster head which needs small amount of transmission energy.

The limitation of LEACH is that the cluster head node consumes more energy as compared to non-cluster head nodes. The failure of cluster-head node breaks the communication of all of its connected nodes.

\section{B. Coverage Configuration Protocol (CCP)}

$\mathrm{CCP}$ is a decentralized protocol. It proposes that every sensor has a static circular covering area, having sensor at center [9]. CCP protocol also proposed that every sensor know the information of its location correctly. For the required coverage degree, CCP protocol configures a network. Various degrees of sensing coverage are needed by many of the applications, in which each location of area is tracked by many nodes. Each sensor node can be in one of the three modes in CCP protocol; active, listen and sleep. Sensor node actively covers the field for an object in an active mode. The node switch off its radio to preserve energy in sleep mode. The sleeping node repeatedly goes into listening mode to check whether to enter into active mode or not. The node remains in an active mode until or unless the $\mathrm{CCP}$ switch off condition is true. Each sensor node in $\mathrm{CCP}$ requires managing a neighborhood table. In this way node can find the coverage overlap to inquire switch off criteria.

The CCP is not energy efficient due to the fact that it consumes more energy with frequent switching of nodes from switch-on to switch-off and vice versa. Moreover, CCP cannot overcome the sensing holes due to low number of active nodes.

\section{Optimal Geographical Density Control (OGDC)}

OGDC is a density control protocol for full coverage. It finds the minimal number of engaging nodes [10]. OGDC makes use of localization methods in which nodes have the information of their exact position [11] [12]. It is also assumed in OGDC that the broadcasting range is at least double the covering range. In this protocol sensors can have one of three modes; OFF, ON and UNDECIDED. The procedure executes in cycles. In the starting of first cycle, all sensors join to choose an active node based on a switch on condition and wake up, adjust their mode to UNDECIDED. The first sensor of every cycle is selected randomly in decentralized way. All nodes change their modes to ON or OFF at the end of the execution of a cycle and still in that mode till starting of the second cycle.

\section{Lightweight Deployment-Aware Scheduling (LDAS)}

The objective of LDAS protocol is to sustain sufficient covering range and maximize the life time of WSN [13]. Among neighboring wireless sensors, the procedure of LDAS protocol evaluates the redundant covering area. As LDAS proposed that sensors have no attach device like GPS to get the information of location, on the probability of average partial and complete redundancy LDAS give tight upper and lower bounds.

\section{E. Obstacle-Resistant Robot Deployment (ORRD)}

The ORRD protocol utilizes one robot in placing static sensors for gaining full coverage with minimum energy consumption and deployment time [14]. The robot node in ORRD consumes less energy and provides better coverage with minimal deployment time. But, the algorithm gets stuck in dead edges with early dropped nodes or obstacles [15].

\section{F. Enhanced Configuration Control Protocol (ECCP)}

The coverage area of a WSN is decreased with the presence of sensing voids [15]. To avoid sensing void the CCP protocol is inadequate. In a sensor network to prevent from sensing 
voids ECCP algorithm gives a method however, additional number of active sensor nodes are required. The working of CCP is enhanced with the addition of a condition that the borders of the targeted area are sensed by neighboring sensors.

Although to maintain the more number of needed active nodes, ECCP protocol make sure that in the region there is no sensing void. It also ensures the full coverage of region. The limitation of ECCP is that additional active sensors involve as compared to CCP due to the ECCP's extra node switch off conditions.

\section{G. Fuzzy Based Priority Coverage (FBPC)}

FBPC coverage protocol is introduced in [16] to enhance the coverage in WSN. The movement of nodes depends upon the obstacles, distance from the borders and number of neighbors. The procedures in the proposed protocol depend on fuzzy inference engines and virtual forces. However, the average moving distance is relatively very long in the proposed protocol which consumes more energy resources.

\section{H. Time Constrained Targets Patrolling (TCTP)}

The TCTP algorithm is proposed, in which every target is designated a weight and moveable sensors which monitor every target according to its weight [17]. The algorithm establishes single path, and make sure that targets having high weight are visited continuously. The impairment in any section of patrol path badly affects the performance of the algorithm. Additionally, to sense all the targets the number of mobile sensors may not be enough.

\section{Random Backoff Sleep Protocol (RBSP)}

RBSP is a search based protocol which uses data regarding the remaining energy level of the on-duty nodes [18]. For the calculation of Backoff Sleep Timer, RBSP applies a novel backoff algorithm. Depending on residual energy (RE) of active node, RBSP protocol makes use of sleeping window in order to select a random value evenly. Through this method, the possibility of a neighboring nodes' switching on status is very down whereas on-duty node has high remaining energy.

In RBSP, when the residual energy of the on-duty node is less than the specified threshold, the neighboring off-duty node wake-up continuously. RBSP minimizes the network lifetime and energy wastage due to the unneeded frequent wake-up of a sleeping node. RBSP makes sure that sufficient numbers of active nodes are available for a long period of time.

\section{J. Discharge Curve Backoff Sleep Protocol (DCBSP)}

The DCBSP protocol provides better coverage established on normalized standard battery discharge curve. This curve is used to find the back-off sleep-time for neighboring sensor nodes which are in sleeping mode [19]. In DCBSP, each sensor has three functioning modes that are likely to ACTIVE, RBSP SLEEP and FLOAT. At start all sensors are in sleeping mode. The sleeping node awakens and switch in to a floating mode upon the expiration of Back-off sleep timer.

DCBSP is a location-unaware and statistical based protocol. To avoid irregular and unwanted continuously wake ups of off-duty sensors, the optimal back-off sleep-time obtained from normalized generic discharged curve. As a result, off-duty nodes activate only near to the deactivation of on-duty nodes.

\section{K. Distributed Lifetime Coverage Optimization (DiLCO)}

DiLCO protocol uses two techniques; network leader selection and scheduling to increase the life cycle of network and sustains coverage [20]. Firstly, the divide and conquer methodology is applied on an area of interest in order to divide it into subareas. DiLCO protocol is periodic protocol in which a period begins with discovery phase to send and receive data among sensors of likely subareas, to select a node (head) to achieve coverage area. Each period consists of four phases namely Leader Election, Information Exchange, Sensing, and Decision. For every period it uses the one cover set for sensing task. Periodic scheduling increases the robustness against the failures of node. In periodic scheduling, a node is excluded from the scheduling process if it is out of energy or fails before taking the decision.

\section{PERFORMANCE COMPARISON}

The performance comparison of above mentioned coverage protocols is based on their coverage degree, sensing area coverage, characteristics, objectives and node scheduling. The comparison is shown in Table 1 .

TABLE I. COMPARATIVE ANALYSIS FOR COVERAGE PROTOCOLS

\begin{tabular}{|l|l|l|l|l|l|l|}
\hline \multirow{2}{*}{ References } & \multirow{2}{*}{ Protocols } & \multicolumn{4}{|c|}{ Parameters } \\
\cline { 3 - 7 } & Coverage Degree & Sensing Area Coverage & Characteristics & Constraint and Objective & Node scheduling \\
\hline$[8]$ & LEACH & $\mathrm{NE}$ & 2-D Circular & Distributed & $\begin{array}{l}\text { As much as possible switching } \\
\text { off non head nodes }\end{array}$ & $\begin{array}{l}\text { Optimized base on } \\
\text { cluster }\end{array}$ \\
\hline$[9]$ & $\mathrm{CCP}$ & $\mathrm{NE}$ & $\mathrm{NE}$ & Distributed & $\begin{array}{l}\text { Configured for guaranteeing } \\
\text { coverage }\end{array}$ & Random \\
\hline$[9-12]$ & OGDC & $\mathrm{Cd}=1$ & $2.12 \%(+)$ & Distributed & $\begin{array}{l}\text { For full coverage finds the least } \\
\text { number of engaging node }\end{array}$ & $\begin{array}{l}\text { Periodic based on } \\
\text { coverage }\end{array}$ \\
\hline$[13]$ & LDAS & $\mathrm{Cd}=1$ & 2-D Circular & Distributed & $\begin{array}{l}\text { To sustain covering area and } \\
\text { maximize the network lifetime }\end{array}$ & Random \\
\hline
\end{tabular}




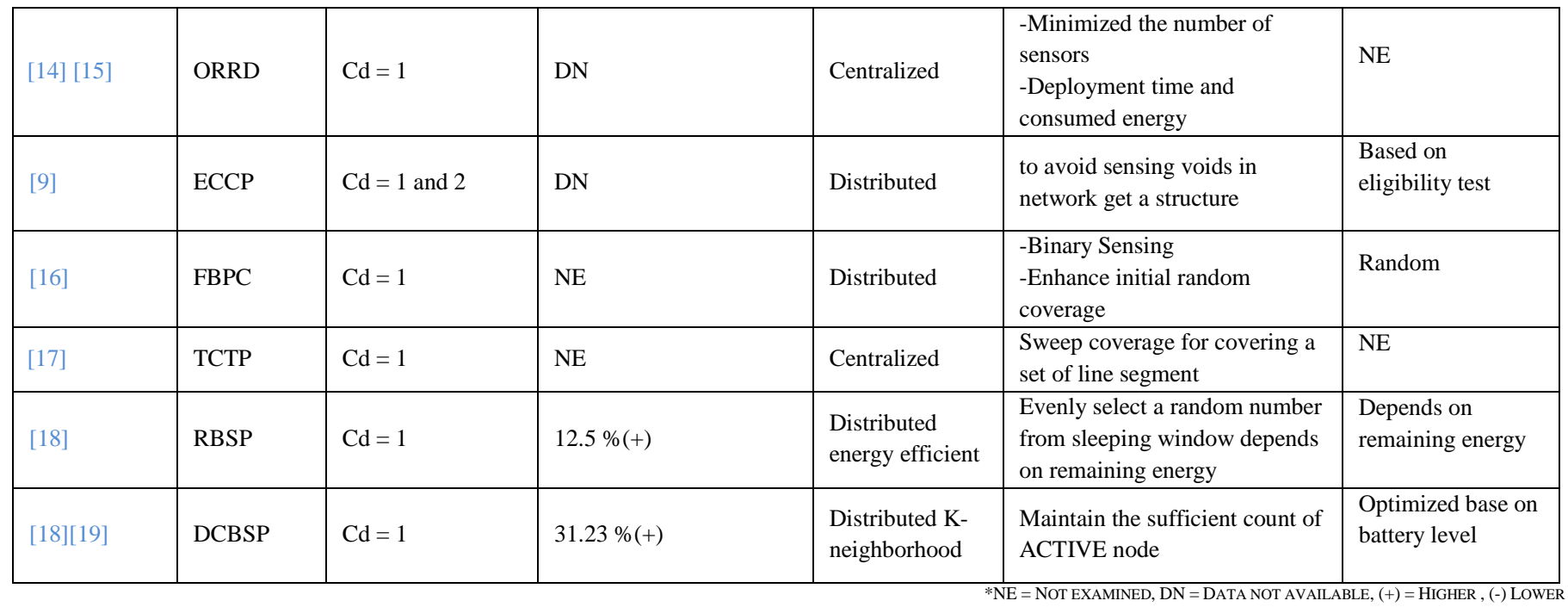

\section{CONCLUSION}

In WSN, coverage redundancy can be minimized by using various types of coverage protocols. Coverage is the important problem in wireless sensor networks (WSNs). Many algorithms have been proposed for the solution of coverage issues during past years. In this review article, we presented a brief overview of coverage optimization protocols. We take coverage optimization protocols along with energy efficiency factors into consideration and explained the various working techniques of both with comparison. It is observed that the basic purpose of coverage protocols is to retain the essential group of working nodes by switching off the redundant nodes in order to save the energy. By minimizing coverage overlap we can attain the energy conservation that maximizes the lifespan of the network.

\section{REFERENCES}

[1] Khalid Mahmood, Muhammad Amir Khan, Mahmood ul Hassan, Ansar Munir Shah, Shahzad Ali, and Muhammad Kashif Saeed, "Intelligent On-Demand Connectivity Restoration for Wireless Sensor Networks," Wireless Communications and Mobile Computing, vol. 2018, Article ID 9702650, 10 pages, 2018. https://doi.org/10.1155/2018/9702650.

[2] Mittal, N., Singh, U., \& Sohi, B. S. (2017). A stable energy efficient clustering protocol for wireless sensor networks. Wireless Networks, 23(6), 1809-1821.

[3] Kong, P., Fang, G., He, C., \& Liu, Z. (2017, July). Topology optimization of port wireless sensor network based on small-world network. In Circuits, System and Simulation (ICCSS), 2017 International Conference on (pp. 157-161). IEEE.

[4] Zhao, J., Yağan, O., \& Gligor, V. (2014, September). Connectivity in secure wireless sensor networks under transmission constraints. In Communication, Control, and Computing (Allerton), 2014 52nd Annual Allerton Conference on (pp. 1294-1301). IEEE.

[5] Mohamed, S. M., Hamza, H. S., \& Saroit, I. A. (2017). Coverage in mobile wireless sensor networks (M-WSN): A survey. Computer Communications, 110, 133-150.

[6] Naranjo, P. G. V., Shojafar, M., Mostafaei, H., Pooranian, Z., \& Baccarelli, E. (2017). P-SEP: a prolong stable election routing algorithm for energy-limited heterogeneous fog-supported wireless sensor networks. The Journal of Supercomputing, 73(2), 733-755.

[7] Bhowmik, S., \& Giri, C. (2013). A novel fuzzy sensing model for sensor nodes in wireless sensor network. In Intelligent Informatics(pp.
365-371). Springer, Berlin, Heidelberg.

[8] Heinzelman, W. R., Chandrakasan, A., \& Balakrishnan, H. (2000, January). Energy-efficient communication protocol for wireless microsensor networks. In System sciences, 2000. Proceedings of the 33rd annual Hawaii international conference on (pp. 10-pp). IEEE.

[9] Zhang, H., \& Hou, J. C. (2005). Maintaining sensing coverage and connectivity in large sensor networks. Ad Hoc \& Sensor Wireless Networks, 1, 14-28.

[10] Ye, F., Zhong, G., Cheng, J., Lu, S., \& Zhang, L. (2003,May). PEAS: A robust energy conserving protocol for long-lived sensor networks. In Distributed computing systems, 2003. Proceedings. 23rd international conference on (pp. 28-37).

[11] Cerpa, A., \& Estrin, D. (2004). ASCENT: Adaptive self- configuring sensor networks topologies. IEEE transactions on mobile computing, $3(3), 272-285$.

[12] Li, J., Andrew, L. L., Foh, C. H., Zukerman, M., \& Chen, H. H. (2009). Connectivity, coverage and placement in wireless sensor networks. Sensors, 9(10), 7664-7693.

[13] Lazos, L., \& Poovendran, R. (2006). Stochastic coverage in heterogeneous sensor networks. ACM Transactions on Sensor Networks (TOSN), 2(3), 325-358.

[14] Chang, C. Y., Chang, C. T., Chen, Y. C., \& Chang, H. R. (2009). Obstacle-resistant deployment algorithms for wireless sensor networks. IEEE Transactions on Vehicular Technology, 58(6), 2925-2941.

[15] Falcon, R., Li, X., \& Nayak, A. (2010, June). Carrier- based coverage augmentation in wireless sensor and robot networks. In Distributed Computing Systems Workshops (ICDCSW), 2010 IEEE 30th International Conference on (pp. 234-239). IEEE.

[16] Farahani, B. J., Ghaffarian, H., \& Fathy, M. (2009). A fuzzy based priority approach in mobile sensor network coverage. International Journal of Recent Trends in Engineering, 2(1).

[17] Chang, C. Y., Yu, G. J., Wang, T. L., \& Lin, C. Y. (2014). Path construction and visit scheduling for targets by using data mules. IEEE Transactions on Systems, Man, and Cybernetics: Systems, 44(10), 12891300.

[18] More, A., \& Raisinghani, V. (2014). Random backoff sleep protocol for energy efficient coverage in wireless sensor networks. In Advanced Computing, Networking and Informatics-Volume 2(pp. 123-131). Springer, Cham.

[19] More, A., \& Raisinghani, V. (2015). Discharge curve backoff sleep protocol for energy efficient coverage in wireless sensor networks. Procedia Computer Science, 57, 1131-1139.

[20] Idrees, A. K., Deschinkel, K., Salomon, M., \& Couturier, R. (2015). Distributed lifetime coverage optimization protocol in wireless sensor networks. The Journal of Supercomputing, 71(12), 4578-4593. 